\title{
Graded-permittivity polymer nanocomposites as superior dielectrics
}

\author{
Tommaso Nardi ${ }^{1}$, Nicolas Mora ${ }^{2}$, Farhad Rachidi $^{2}$, Yves Leterrier $^{1 *}$ \\ ${ }^{1}$ Laboratoire de Technologie des Composites et Polymères (LTC), Ecole Polytechnique \\ Fédérale de Lausanne (EPFL), CH-1015 Lausanne, Switzerland \\ ${ }^{2}$ Electromagnetic Compatibility Laboratory (EMC-Lab), Ecole Polytechnique Fédérale de \\ Lausanne (EPFL), CH-1015 Lausanne, Switzerland \\ (*) yves.leterrier@epfl.ch
}

Abstract. Materials exhibiting a gradient in permittivity are synthesized through the application of an external magnetic field to a suspension of $\mathrm{Fe}_{3} \mathrm{O}_{4} @ \mathrm{TiO}_{2}$ nanoparticles in an epoxy matrix. It is shown how the application of the magnetic field not only induces the magnetophoretic motion of the particles, but causes also their alignment in high aspect ratio structures. The combination of these two effects gives rise to graded nanocomposites exhibiting gradients in permittivity that go beyond the ones predicted for nanocomposites with homogeneously distributed and isotropic inclusions. Moreover, it is demonstrated through numerical simulations how such nanocomposites, employed as electrical insulators, can efficiently reduce the electrical field stress at the interface between the electrode and the insulator, being therefore suitable candidates as long-lasting high-voltage dielectrics.

Keywords: Electrical properties, Functional composites, Nano particles

\section{Introduction}

For size reduction and enhanced reliability of electric power equipment, the electric field 
stress around solid insulators must be considered [1]. In the case of high-voltage insulators, the insulation performance around the insulator is improved by various techniques, such as controlling the insulator shape, adding shield electrodes for electric field relaxation, or through the introduction of an embedded electrode [2]. However, these electric field management techniques lead to complicated equipment structures and increased manufacturing cost. Thus, new concepts for solid insulators emerge, which are should in principle overcome the drawbacks associated with complex configurations. Kurimoto et al. [2, 3] investigated the applicability of functionally graded materials (FGM) with spatial distribution of dielectric permittivity $\varepsilon(\varepsilon-\mathrm{FGM})$ to reduce electric stresses on the electrode surface in contact with solid insulating materials. Such stresses are among the key factors, which control the long-term performance of high-voltage insulators. Their results show that the lifetime of $\varepsilon$-FGM insulators based on epoxy and graded concentrations of $\mathrm{TiO}_{2}$ and $\mathrm{Al}_{2} \mathrm{O}_{3}$ was up to 8.2 times higher than that of a homogeneous insulator. Previous work already showed how polymer composite FGM were efficiently synthesized through the application of an external magnetic field to a suspension of magnetic nanoparticles in a polymer matrix, prior to polymerization [4-6]. This synthetic strategy is employed in this work in order to create FGM exhibiting gradients in electrical permittivity.

First, we synthesized $\mathrm{Fe}_{3} \mathrm{O}_{4} @ \mathrm{TiO}_{2}$ nanoparticles (NP) with an amorphous $\mathrm{TiO}_{2}$ shell and we increased their permittivity by inducing the partial crystallization of the shell through a calcination step. Hence, we created graded permittivity nanocomposites through the magnetophorese of high dielectric constant and magnetic $\mathrm{Fe}_{3} \mathrm{O}_{4} @ \mathrm{TiO}_{2} \mathrm{NP}$ in a low-viscosity epoxy matrix. Experimental characterizations and numerical simulations demonstrated how such nanocomposites have the potential to efficiently reduce the electrical field stress at the electrode-insulator interface and deserve good attention for applications as long-lasting highvoltage electrical insulators. 


\section{Experimental}

Materials: Titanium(IV) chloride (99.9\%) was purchased from Acros. Hydrogen peroxide (30\%) was purchased from Reactolab SA. The epoxy resin DER 321 was purchased from Dow whereas the hardener diethylenetriamine was purchased from Aldrich. Ammonia solution (25\% min) was purchased from VWR. All products were used as received without any further purification.

Synthesis of $\mathrm{Fe}_{3} \mathrm{O}_{4} @ \mathrm{TiO}_{2}$ core-shell nanoparticles: To synthesize the $\mathrm{Fe}_{3} \mathrm{O}_{4}$ cores, the synthetic procedure reported by Bumb et al. was followed [7]. Briefly, $\mathrm{FeCl}_{3} \cdot 6 \mathrm{H}_{2} \mathrm{O}(8 \mathrm{mmol})$ and $\mathrm{FeCl}_{2} \cdot 4 \mathrm{H}_{2} \mathrm{O}(4 \mathrm{mmol})$ were dissolved in deionized water $(190 \mathrm{ml})$ at room temperature by magnetic stirring in a beaker. Under conditions of vigorous stirring, $25 \% \mathrm{NH}_{3}(10 \mathrm{ml})$ was poured down the vortex of the iron solution. Immediately, magnetite formed a black precipitate. After stirring for 10 minutes, the particles were centrifuged and dispersed in of deionized water $(50 \mathrm{ml})$. The as synthesized $\mathrm{Fe}_{3} \mathrm{O}_{4} \mathrm{NP}$ were subsequently coated exploiting a synthetic procedure for $\mathrm{TiO}_{2}$ nanoparticles reported by Buscaglia et al. [8] with minor modifications. First, $\mathrm{TiCl}_{4}(0.7 \mathrm{ml})$ were added to the ice-cooled solution of $\mathrm{Fe}_{3} \mathrm{O}_{4}$ nanoparticles in water $(50 \mathrm{ml})$. Then, a peroxotitanium complex was prepared by adding $\mathrm{H}_{2} \mathrm{O}_{2}$ $(30 \%, 1 \mathrm{ml})$ to the above-mentioned $\mathrm{Fe}_{3} \mathrm{O}_{4} / \mathrm{TiCl}_{4}$ solution. The $\mathrm{pH}$ was hence increased through the addition of of aqueous ammonia (4.5 ml, $5.44 \mathrm{~mol} / \mathrm{L})$ and the solution was slowly heated to $95^{\circ} \mathrm{C}$ and maintained at this temperature for 5 hours. Finally, the solution was let cool down to room temperature and particles were washed and collected by centrifugation. To increase the permittivity of the as-synthesized particles, a calcination step at $450^{\circ} \mathrm{C}$ for 6 hours under $\mathrm{N}_{2}$ flow $(10 \mathrm{ml} / \mathrm{min})$ was performed.

Characterization: The NP morphology was characterized using a Philips/FEI CM12 transmission electron microscope (TEM) at an accelerating voltage of $120 \mathrm{kV}$. Typically, few 
drops of diluted NP suspensions (in cyclohexane for $\mathrm{Fe}_{3} \mathrm{O}_{4} \mathrm{NP}$, in ethanol for $\mathrm{Fe}_{3} \mathrm{O}_{4} @ \mathrm{TiO}_{2}$ NP) were deposited on carbon filmed copper grids (200 mesh, Plano GmbH). Fourier Transform Infrared (FTIR) spectra were acquired using a Perkin Elmer Spectrum One MIR $\left(600-4000 \mathrm{~cm}^{-1}\right)$ with an attenuated total reflectance (ATR) accessory. The spectra were acquired with 32 scans and a resolution of $4 \mathrm{~cm}^{-1}$. Magnetization loops of $\mathrm{Fe}_{3} \mathrm{O}_{4} @ \mathrm{TiO}_{2}$ coreshell NP in powder form were measured at $\mathrm{T}=295 \mathrm{~K}$ by means of a Vibrating Sample magnetometer (VSM) operating in the $\pm 15 \mathrm{kOe}$ field range and equipped with a liquid $-\mathrm{N}_{2}$ continuous-flow cryostat. X-ray powder diffraction (XRPD) data for qualitative and quantitative phase analysis were collected using a Bragg-Brentano $\theta-2 \theta$ diffractometer (Philips PW1729 PANalytical, Netherlands) equipped with a gas proportional detector. The radiation source was an X-ray tube with copper radiation $\left(\lambda \mathrm{Cu} \mathrm{K} \mathrm{K}_{\alpha 1}=1.54059 \AA\right)$ and the anode tube load was $40 \mathrm{kV}$ and $35 \mathrm{~mA}$. The samples were loaded on a quartz flat holder in order to have a zero background. XRPD patterns, both for the phase composition and the structural characterization, were collected at room temperature in the $15-135^{\circ} 2 \theta$ range, with a scanning rate of $0.004^{\circ} \mathrm{s}^{-1}$ and a step size of $0.02^{\circ} 2 \theta$. Preliminary qualitative phase analyses were performed using the X'Pert High Score Plus software (PANalytical, Netherlands). In order to determine the amorphous phase content of each powder, pure $\alpha-\mathrm{Fe}_{2} \mathrm{O}_{3}$ was chosen as internal standard. Mixed samples were prepared diluting original samples with $10 \mathrm{wt} \%$ of hematite. The internal standard hematite $\left(\alpha-\mathrm{Fe}_{2} \mathrm{O}_{3}\right)$ was refined against the standard reference Si powder 640c (NIST, USA) using a powder pattern collected by spiking hematite with ca. $10 \mathrm{wt} \% \mathrm{Si}$. The concentration gradients were investigated analyzing the Ti content by scanning electron microscopy with energy dispersive X-ray spectroscopy (SEM-EDX, Philips FEI XLF-30 FEG) at an accelerating voltage $\mathrm{V}_{\text {acc }}$ of $13 \mathrm{kV}$ and at a constant working distance $(11 \mathrm{~mm})$ and spot size (4). In order to correlate the SEM-EDX signal intensity with the actual nanoparticle content, a calibration curve was created by performing an EDX 
analysis on homogeneous samples containing $0,2,6$ and 12 vol\% of nanoparticles. The volume fraction of nanoparticles in the homogeneous nanocomposites was calculated by thermogravimetric analysis and by converting the resulting nanoparticle weight fraction into a volume fraction using the densities of the epoxy $\left(1.16 \mathrm{~g} / \mathrm{cm}^{3}\right), \mathrm{Fe}_{3} \mathrm{O}_{4}\left(5.21 \mathrm{~g} / \mathrm{cm}^{3}\right)$ and $\mathrm{TiO}_{2}$ $\left(4.23 \mathrm{~g} / \mathrm{cm}^{3}\right)$. Measurements of the complex effective permittivity of the composites were performed using an impedance-material analyzer Agilent E4491A, a permittivity test fixture Agilent 16453A (in the frequency band $1 \mathrm{MHz}$ to $1 \mathrm{GHz}$ ) and using a dielectric probe Agilent 85070D.

\section{Results and Discussion}

\subsection{Analysis of the $\mathrm{Fe}_{3} \mathrm{O}_{4} @ \mathrm{TiO}_{2}$ nanoparticles}

Figure 1 regroups structural and magnetic analyses of the synthesized NP. TEM observations indicated that dimensions were in the order of $100 \mathrm{~nm}$ (Figure 1a) and that they did not change after the calcination step (Figure $1 \mathrm{~b}$ ). Moreover, many $\mathrm{Fe}_{3} \mathrm{O}_{4}$ cores were included in the same $\mathrm{TiO}_{2}$ shell. The FTIR spectra of the particles before and after the calcination step evidence notable differences (Figure 1c). While both present a peak at low wavenumbers (below $800 \mathrm{~cm}^{-1}$ ) due to $v \mathrm{Ti}-\mathrm{O}-\mathrm{Ti}$ vibrations [9], signals deriving from the stretching vibrations of different hydroxyl groups, such as those due to adsorbed water (between 3300 and $3500 \mathrm{~cm}^{-1}$ ), or from the deformation modes of adsorbed $\mathrm{NH}_{3}$ (between 1700 and 1450 $\mathrm{cm}^{-1}$ ), are visible only in the spectrum recorded for $\mathrm{Fe}_{3} \mathrm{O}_{4} @ \mathrm{TiO}_{2} \mathrm{NP}$ before calcination. This suggests that the calcination step induced the desorption of the adsorbed water and ammonia from the surface of the particles. A high-field magnetization of $52 \mathrm{emu} / \mathrm{g}$ is observed for bare $\mathrm{Fe}_{3} \mathrm{O}_{4}$ nanoparticles, still well below the saturation magnetization of bulk magnetite (92 
emu/g) [10] but above the value found for the magnetite NP synthesized through a high temperature procedure $(27 \mathrm{emu} / \mathrm{g})[11]$. Both curves of $\mathrm{Fe}_{3} \mathrm{O}_{4} @ \mathrm{TiO}_{2} \mathrm{NP}_{\text {before and after }}$ calcination are characterized by the absence of magnetic hysteresis and by a saturating behavior at high fields. The high-field magnetization drops from $16 \mathrm{emu} / \mathrm{g}$ to $11.3 \mathrm{emu} / \mathrm{g}$ after calcination. This is due to the well-studied phase transformation of magnetite to the less magnetic but more thermodynamically stable oxidized forms of iron oxide, that is maghemite $\left(\gamma-\mathrm{Fe}_{2} \mathrm{O}_{3}\right)$ and hematite $\left(\alpha-\mathrm{Fe}_{2} \mathrm{O}_{3}\right) \cdot[12]$

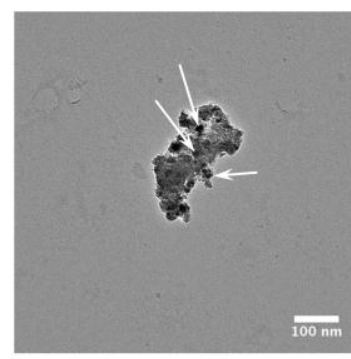

(a)

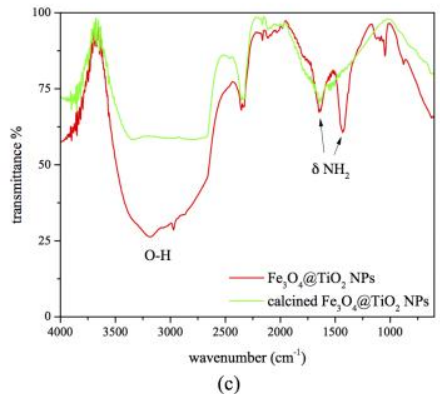

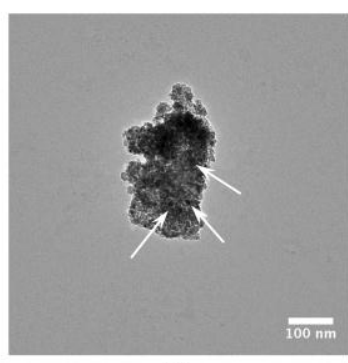

(b)

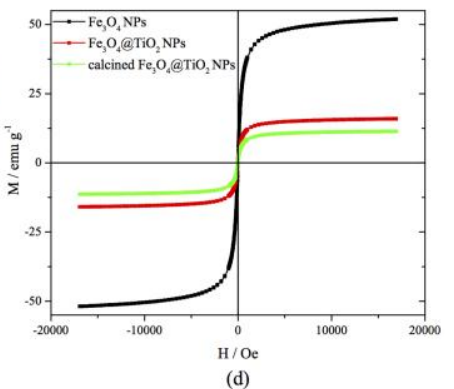

Figure 1. TEM images of individual $\mathrm{Fe}_{3} \mathrm{O}_{4} @ \mathrm{TiO}_{2}$ nanoparticles with arrows pointing to several $\mathrm{Fe}_{3} \mathrm{O}_{4}$ cores before (a) and after (b) the calcination step $\left(450^{\circ} \mathrm{C}\right.$ for 6 hours), FTIR spectra of the nanoparticles before (red) and after (green) the calcination step (c) and room temperature magnetization curves of bare magnetite nanoparticles and $\mathrm{Fe}_{3} \mathrm{O}_{4} @ \mathrm{TiO}_{2}$ nanoparticles before and after the calcination step (d).

The Rietveld quantitative phase analysis (Figure 2) performed on $\mathrm{Fe}_{3} \mathrm{O}_{4} @ \mathrm{TiO}_{2} \mathrm{NP}$ before and after calcination evidenced how this step induced a partial crystallization of the $\mathrm{TiO}_{2}$ phase, which went from being completely amorphous to exhibiting $\approx 35 \mathrm{wt} \%$ of anatase

(Table 1). Based on the results of this analysis, the dielectric constant $\varepsilon_{r}$ of the $\mathrm{Fe}_{3} \mathrm{O}_{4} @ \mathrm{TiO}_{2}$ NP before and after the calcination step was estimated to be equal to 21 and 31 , respectively, 
using a rule-of-mixture [13] (with $\varepsilon_{\text {anatase }}=50$ [13], $\varepsilon_{\text {amorphous }}=15$ and $\varepsilon_{\text {magnetite }}=10$ [14] where $\varepsilon_{\text {amorphous }}$ was calculated by averaging the values of the dielectric constant for amorphous $\mathrm{TiO}_{2}$, assumed to be equal to 20 [15] and for amorphous $\mathrm{Fe}_{3} \mathrm{O}_{4}$, assumed to be equal to 10 [14]). In light of the results of the magnetic and diffractometric analyses, the execution of a calcination step at $450^{\circ} \mathrm{C}$ for 6 hours was considered a good compromise between the reduction in magnetization and the increment in permittivity.

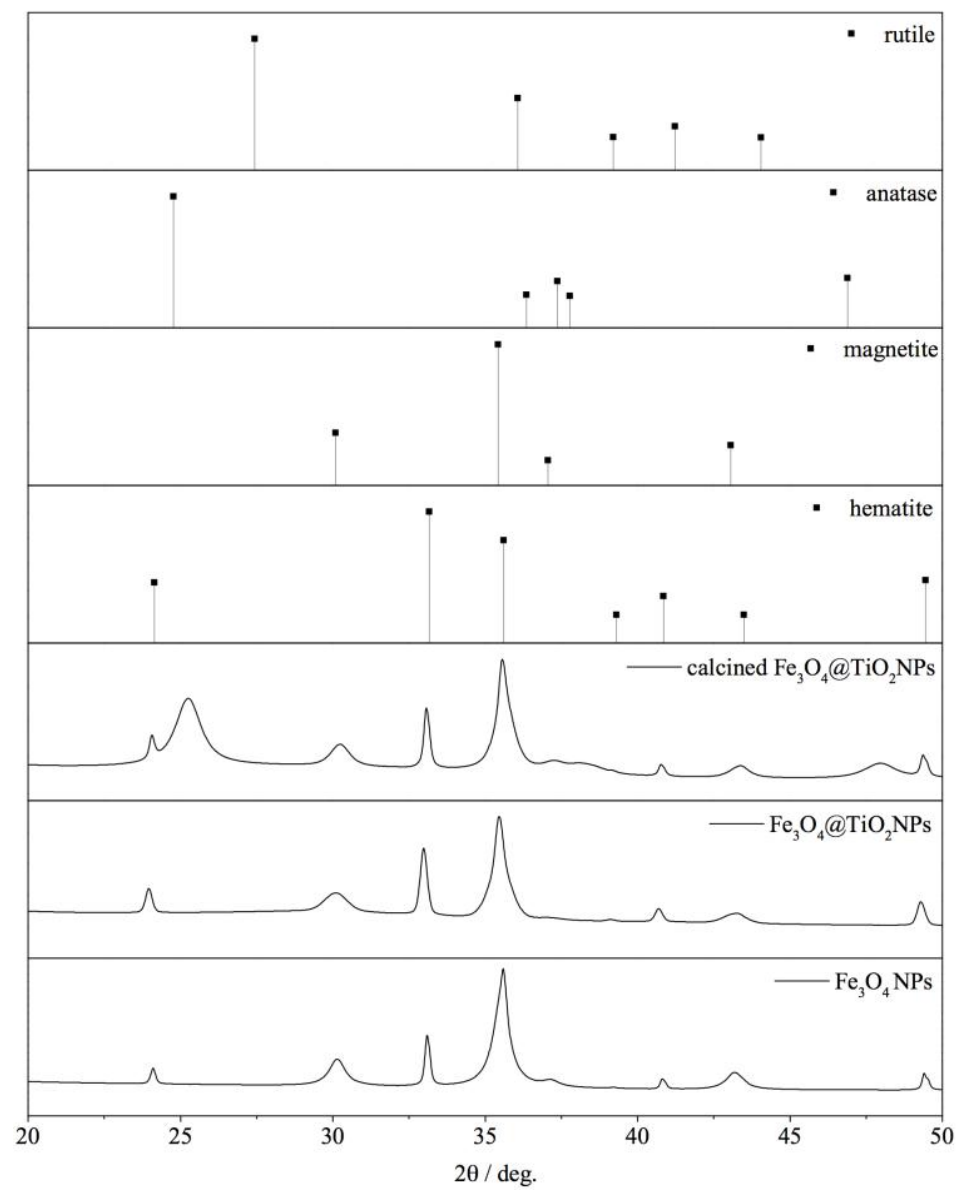

Figure 2. XRD plots of the samples and reference lines of rutile, anatase, magnetite and hematite.

\subsection{Gradient morphology}

Figure 3 shows the complex viscosity of the newtonian epoxy resin and suspensions of $\mathrm{Fe}_{3} \mathrm{O}_{4} @ \mathrm{TiO}_{2}-\mathrm{C} \mathrm{NP}$, which exhibit an increasing shear thinning behavior with increasing NP 
concentration. These suspensions were subjected to the application of the external magnetic field gradient generated by permanent block magnets. In order to minimize processing time, the magnetophorese and curing processes were carried out simultaneously at $60^{\circ} \mathrm{C}$ for 2 hours.

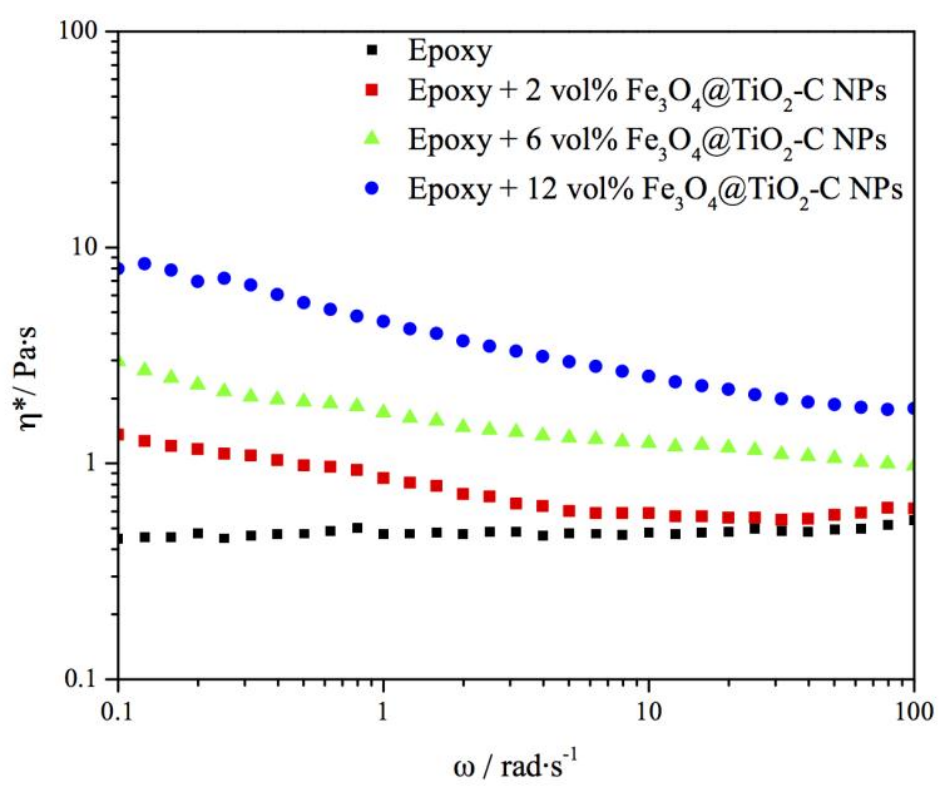

Figure 3. Complex viscosity $\eta^{*}$ of pure epoxy and calcined $\mathrm{Fe}_{3} \mathrm{O}_{4} @ \mathrm{TiO}_{2}$ nanoparticles suspensions in epoxy as a function of frequency and particle fraction as indicated.

The concentration gradients developed during this dual process are shown in Figures 4 and 5. Figure 5 also includes the permittivity of the materials, as detailed in the following section. Notice that the length scales of z-axes in both figures correspond to the actual thickness of the tested samples, ranging from $1.3 \mathrm{~mm}$ to $2.3 \mathrm{~mm}$. It is evident a NP concentration gradient ranging from 1.5 to $6 \mathrm{vol} \%$ developed when the nanocomposite containing $2 \mathrm{vol} \%$ of $\mathrm{Fe}_{3} \mathrm{O}_{4} @ \mathrm{TiO}_{2} \mathrm{NP}$ was subjected to the magnetic field gradient generated by two magnets in repulsion configuration (Figures $4 \mathrm{a} 1-\mathrm{a} 2$ and $5 \mathrm{~b}$ ). An even steeper NP gradient, ranging from 3.5 to $13 \mathrm{vol} \%$, developed instead when the same magnetic field gradient was applied to the suspension containing 6 vol\% of nanoparticles (Figures 4b1-b2 and 5c). A smoother and linear gradient ranging from 9 to $15 \mathrm{vol} \%$ (Figures $4 \mathrm{c} 1-\mathrm{c} 2$ and $5 \mathrm{~d}$ ) was generated in the composite with an initial 12 vol\% of $\mathrm{Fe}_{3} \mathrm{O}_{4} @ \mathrm{TiO}_{2} \mathrm{NP}$. 


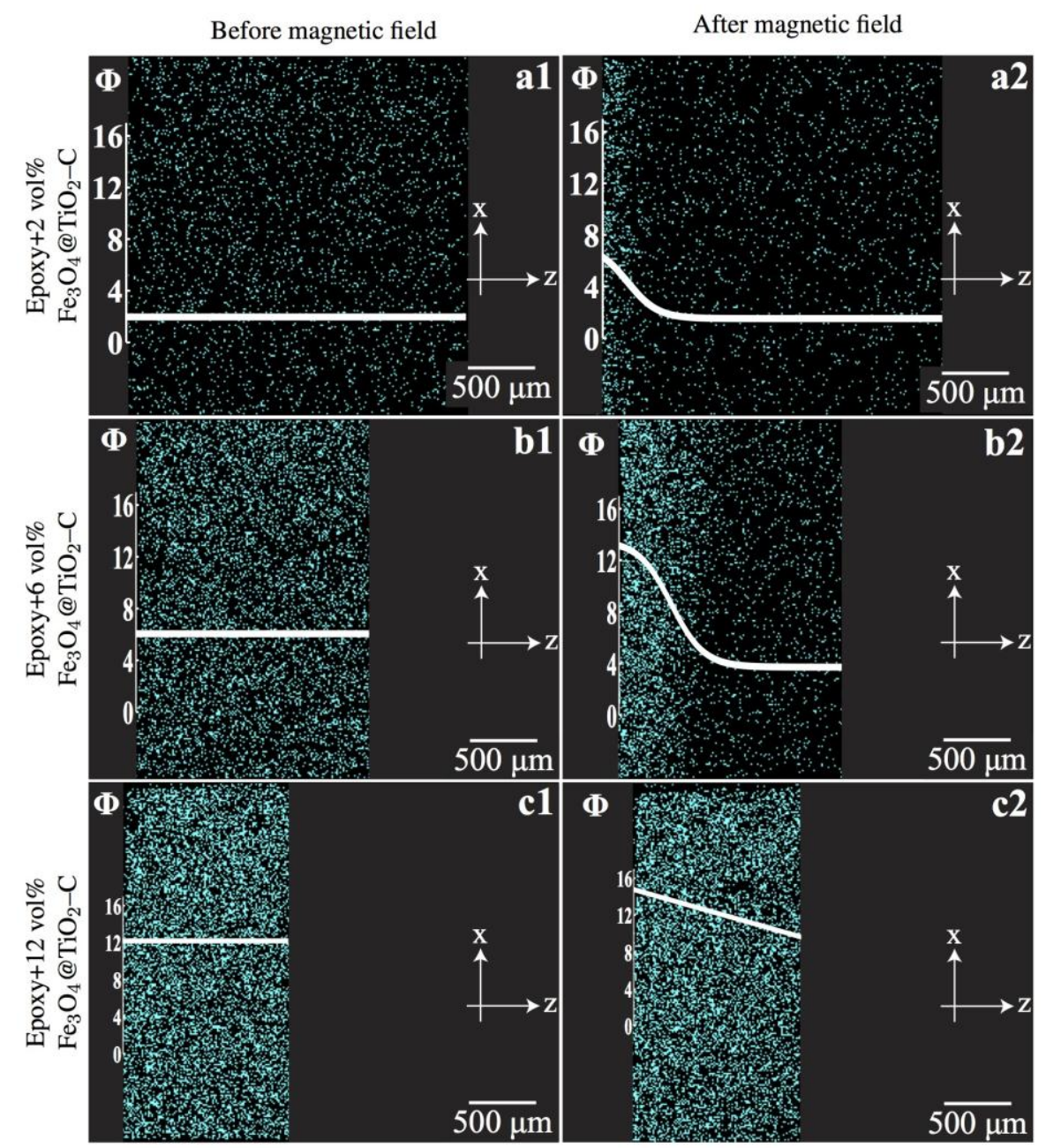

Figure 4. Cross-sectional SEM-EDX spectral images showing the Ti content of different samples having been under the influence of the magnetic force generated by two magnets in repulsion configuration. The superimposed curves indicate the nanoparticle volume fractions all along the sample cross-sections.

\subsection{Permittivity analysis}

The relative permittivity of the pure polymer, homogeneous and graded nanocomposites is reported in Figure 5. The graded samples were sliced and the permittivity $\varepsilon_{r}$ of each slice was measured separately. Each slice had a thickness between $\approx 200$ and $\approx 500 \mu \mathrm{m}$ (represented in grey in Figure 5b-c-d, whereas the white space in between different slices is due to the thickness of the saw). The relative permittivity of the epoxy was 3.52, which marginally increased with the inclusion of 2 vol\% of NP. On the contrary, the creation of a concentration 
gradient led to the formation of a corresponding gradient in permittivity, ranging from 3.36 (for the least concentrated slice) to 5.19 (for the most concentrated slice, Figure 5b). Steep permittivity gradients were achieved when the magnetic field was applied to suspensions containing $6 \mathrm{vol} \%$ and $12 \mathrm{vol} \%$ of $\mathrm{Fe}_{3} \mathrm{O}_{4} @ \mathrm{TiO}_{2}$ nanoparticles. In the first case $\varepsilon_{r}$ ranged from 3.48 to 14.3 (Figure 5c), in the second from 16.4 to 19.4 (Figure 5d). A comparison between the $\varepsilon_{r}$ values of the slices with lowest NP concentration and of the pure epoxy evidenced how a certain concentration threshold had to be attained in order to generate a noticeable increase of the permittivity.
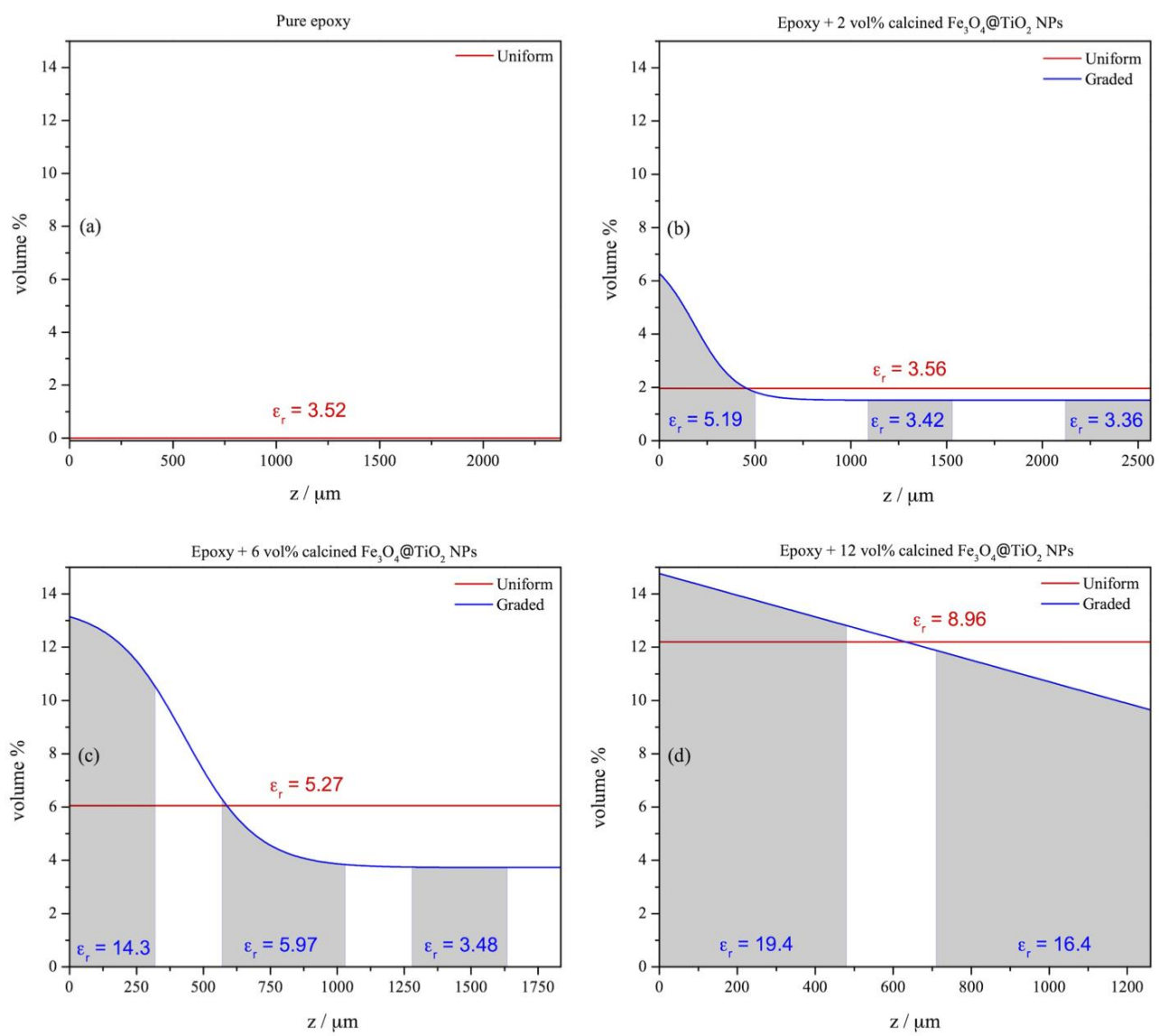

Figure 5. Cross-sectional concentration (vol\%) of calcined $\mathrm{Fe}_{3} \mathrm{O}_{4} @ \mathrm{TiO}_{2}$ nanoparticle before and after the application of the magnetic field gradient, for systems containing an initial concentration of nanoparticles of (a) 0\% (pure epoxy), (b) 2 vol\%, (c) $6 \mathrm{vol} \%$ and (d) 12 vol\%. The permittivity values (at $\mathrm{f}=1 \mathrm{kHz}$ ) of all materials are indicated in the figures (for the graded composites, the permittivity was measured on sample slices, represented as grey areas). 
To underline the goodness of the calcination process for the increase of the NP permittivity, it is worth to mention that the permittivity of homogeneous samples filled with 12 vol\% of noncalcined $\mathrm{Fe}_{3} \mathrm{O}_{4} @ \mathrm{TiO}_{2}$ was equal to 5.92, substantially lower than that of the samples containing the same amount of calcined inclusions $\left(\varepsilon_{r}=8.96\right)$.

The selection of the most appropriate model able to reproduce the experimental permittivity gradients was carried out in light of SEM observations done on the nanocomposites before and after the application of the magnetic field. Indeed, these analyses pointed out how the application of the external magnetic force did not only lead to the magnetophorese of the particles in the direction of an increasing magnetic field, but caused also their alignment in high aspect-ratio structures (Figure 6). Moreover, the alignment resulted to be more evident at increasing NP fractions and where the applied magnetic force was stronger, i.e. in proximity of the magnet. Widely used models for the estimation of the permittivity in composite materials are those developed by Maxwell-Garnett [16] and Bruggeman [17]. However, these models are applicable below the percolation threshold. On the contrary, McLachlan's model can be applied even at higher nanoparticle concentrations and it explicitly includes the percolation threshold as a function of the aspect ratio of the inclusions [18-20]. As such, it is more suitable to be employed for the analysis of our systems, characterized by fillers with variable aspect ratios (depending on the intensity of the applied magnetic field) and variable concentrations (depending on the position along the sample). McLachlan's model reads:

$$
(1-\phi) \frac{\left(\varepsilon_{m}^{1 / s}-\varepsilon_{r}^{1 / s}\right)}{\varepsilon_{m}^{1 / s}+\left(\left(1-p_{c}\right) / p_{c}\right) \varepsilon_{r}^{1 / s}}+\phi \frac{\left(\varepsilon_{m}^{1 / t}-\varepsilon_{r}^{1 / t}\right)}{\varepsilon_{p}^{1 / t}+\left(\left(1-p_{c}\right) / p_{c}\right) \varepsilon_{r}^{1 / t}}=0
$$


Epoxy+2 vol\% $\mathrm{Fe}_{3} \mathrm{O}_{4} @ \mathrm{TiO}_{2}-\mathrm{C}$
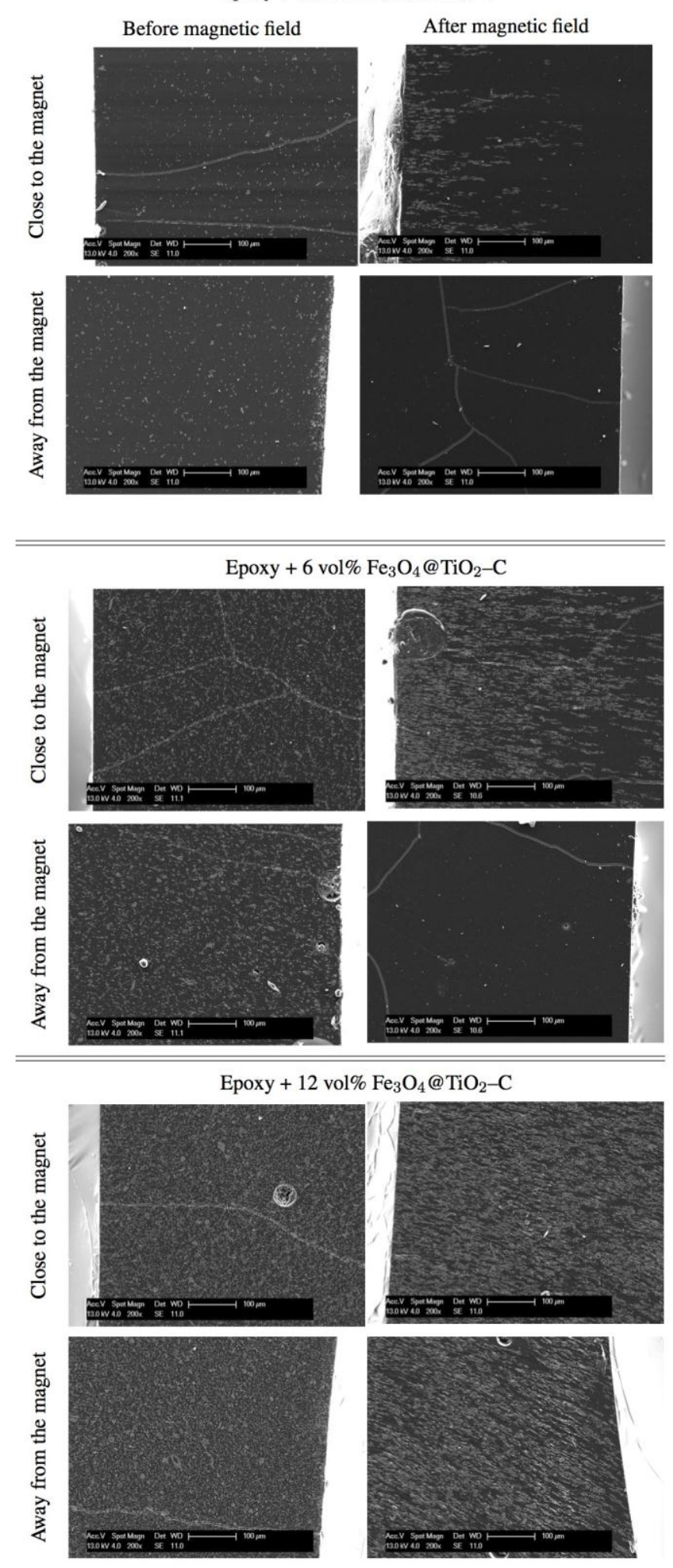

Figure 6. Cross-sectional electron micrographs of homogeneous and graded nanocomposites filled with, from top to bottom, 2 vol\%, 6 vol\% and 12 vol\% of calcinated $\mathrm{Fe}_{3} \mathrm{O}_{4} @ \mathrm{TiO}_{2}$ nanoparticles. In all cases the formation of chains of nanoparticles upon the application of the magnetic field is evident. The surface cracks were produced during SEM sample preparation. 
where $\phi$ is the particle volume fraction, $p_{c}$ is the percolation threshold of inclusions (defined as $1 / a$, where $a$ is the aspect ratio of inclusions [21]), $\varepsilon_{r}$ is the permittivity of the composite and $\varepsilon_{p}$ and $\varepsilon_{m}$ are the permittivities of the particles and of the polymer matrix, respectively [22]. Moreover, the two exponents $s$ and $t$ are empirical parameters. Although explicitly including the percolation threshold $p_{\mathrm{c}}$ and the two exponents $s$ and $t$, McLachlan's effective medium theory does not specify in advance what the values of these parameters should be. As such, the model is suited only to fitting experimental data [19]. In order to find the values for the exponents $s$ and $t$, McLachlan's model was used to fit the experimental permittivity of the homogeneous nanocomposites (Figure 7), resulting in values of $s$ and $t$ equal to 8 and 1 , respectively, while the percolation threshold $p_{c}$, and hence the aspect ratio $a$, were kept equal to 1 .

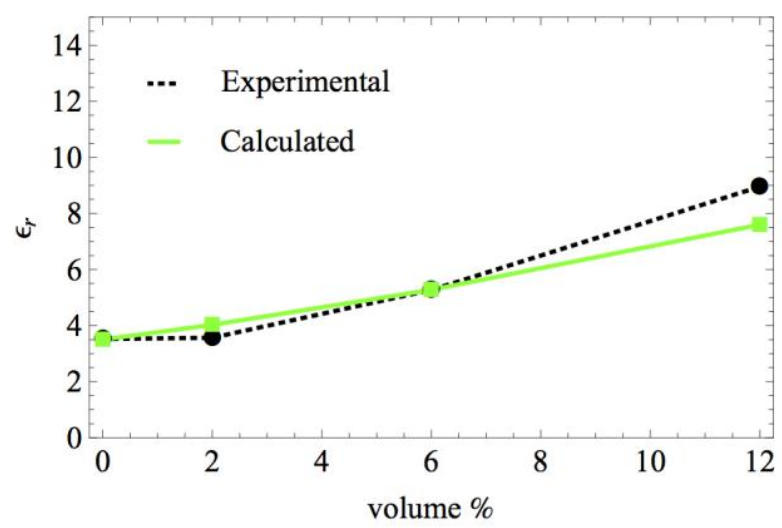

Figure 7. Experimental and calculated (according to Equation 1, with $s=8, t=1$ and $p_{c}=a=1$ ) relative permittivity $\varepsilon_{r}$ of the composite as a function of the calcined $\mathrm{Fe}_{3} \mathrm{O}_{4} @ \mathrm{TiO}_{2}$ nanoparticles volume fraction.

The permittivity of the graded nanocomposites was subsequently calculated using McLachlan's model, based on the known NP concentration gradient (Figure 4 and 5), the values of the exponents determined for the homogeneous composites, and varying the aspect ratio $a$ (and therefore the percolation threshold $p_{c}=1 / a$ ) in order to find the best fit for the experimental data. As shown in Figure 8, for the graded composite containing 2 vol\% of 
$\mathrm{Fe}_{3} \mathrm{O}_{4} @ \mathrm{TiO}_{2} \mathrm{NP}$, the best fit was obtained by setting $a$ equal to 1 (Figure 8a). Even though

SEM observations seem to indicate the formation of high-aspect ratio structures in the proximity of the magnet (Figure 6), the relatively low nanoparticle concentration did not lead
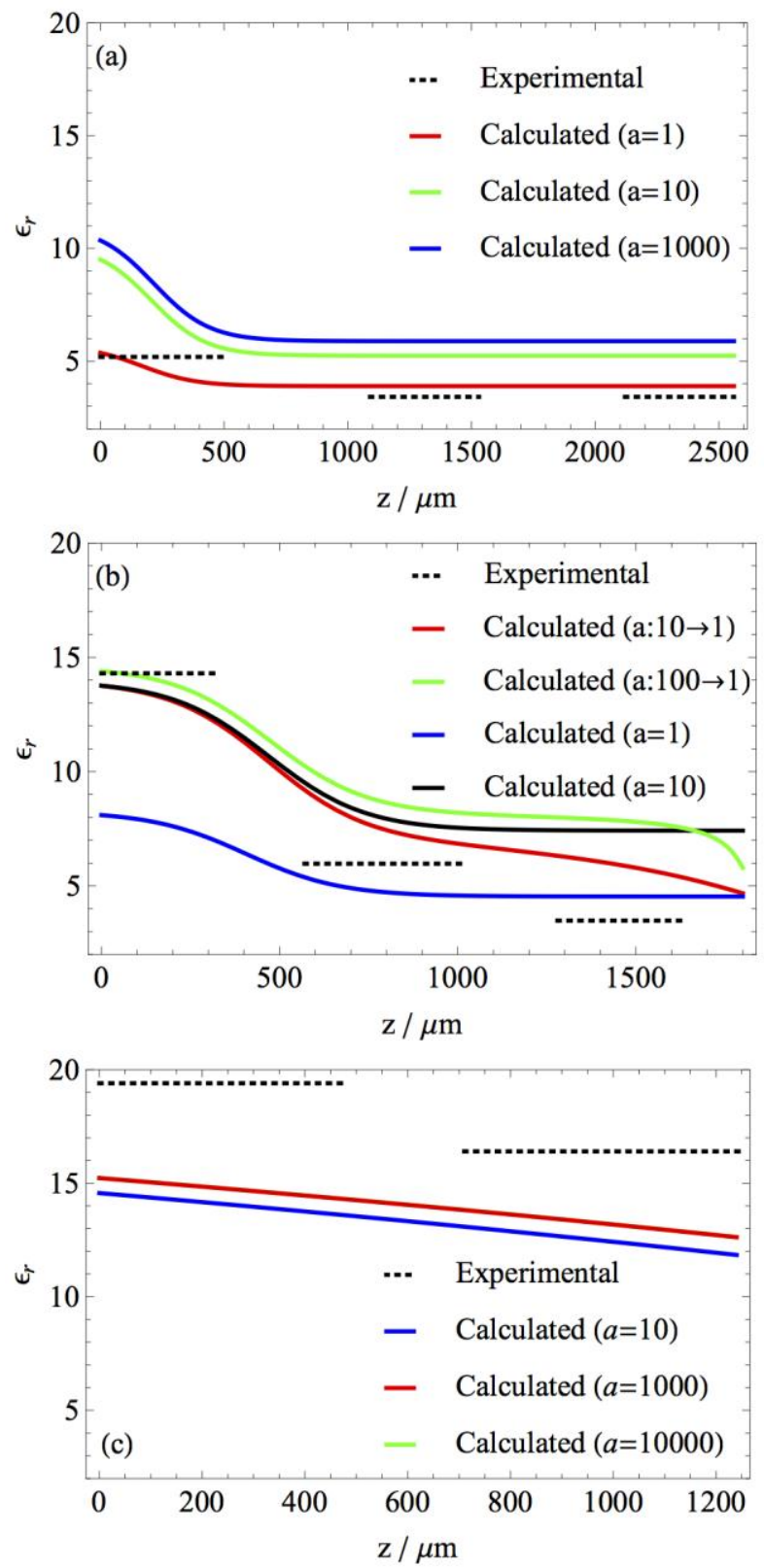

Figure 8. Experimental and calculated (according to Equation 1, with $s=8, t=1$ ) relative permittivity $\varepsilon_{r}$ of graded nanocomposites with an initial concentration of calcined $\mathrm{Fe}_{3} \mathrm{O}_{4} @ \mathrm{TiO}_{2}$ nanoparticles of (a) 2 vol\%, (b) 6 vol\% and (c) $12 \mathrm{vol} \%$ as a function of the position $\mathrm{z}$ along the thickness of the samples and for different values of the aspect ratio $a$, as indicated in the legend. The indication a: $\rightarrow 1$ in (b) means that $a$ varies linearly from $\mathrm{x}$ at $\mathrm{z}=0$ $\mu \mathrm{m}$ to 1 at $\mathrm{z}=1830 \mu \mathrm{m}$. Permittivity trends for $a=1000$ and $a=10000$ in (c) overlap. 
to a percolated system characterized by interconnected particles. For the systems filled with 6 vol\% of $\mathrm{Fe}_{3} \mathrm{O}_{4} @ \mathrm{TiO}_{2} \mathrm{NP}$, the best fit (Figure $8 \mathrm{~b}$ ) was obtained by letting the aspect ratio $a$ vary linearly from 10 (at the most concentrated side) to 1 (at the least concentrated side), which seems to be consistent with the nanocomposite morphology shown in Figure 6. Finally, the results of the composite containing 12 vol\% of $\mathrm{Fe}_{3} \mathrm{O}_{4} @ \mathrm{TiO}_{2}$ nanoparticles were modeled by keeping a constant and equal to 1000 (Figure 8c). Although not as good as the fit obtained for lower nanoparticle volume fraction, the prediction of the composite permittivity follows the experimental trend, and the assumption to assign such a high $a$ value gains credit in light of the SEM observations (Figure 6), which point out the very high nanoparticle alignment all along the thickness of the sample.

\subsection{Computation of the electric field strength}

In order to evaluate their performance in reducing the electric field strength in high-voltage apparatuses, numerical simulations with the finite element method (FEM) solver of the electrostatic module of CST Studio Suite 2015 were carried out. An axisymmetric assembly (Figure 9) comprising two perfectly conducting cylindrical electrodes (diameter $20 \mathrm{~mm}$, thickness $10 \mathrm{~mm}$ ) and a cylindrical insulator (diameter $10 \mathrm{~mm}$, thickness $2 \mathrm{~mm}$ ) was modeled. The homogeneous composites were modeled as dielectric cylinders with a uniform permittivity, whereas graded composites were modeled by stacking together 45 layers with permittivity computed using McLachlan's model and shown in Figure 8. The high-voltage (HV) electrode was declared at fixed potential $\mathrm{V}=100 \mathrm{kV}$ whereas the grounding (GND) electrode was fixed at $\mathrm{V}=0 \mathrm{~V}$. Hence, the electric field distribution along the surface of $\mathrm{HV}$ and GND electrodes (Path \#1 and \#2 in Figure 9) was investigated. 


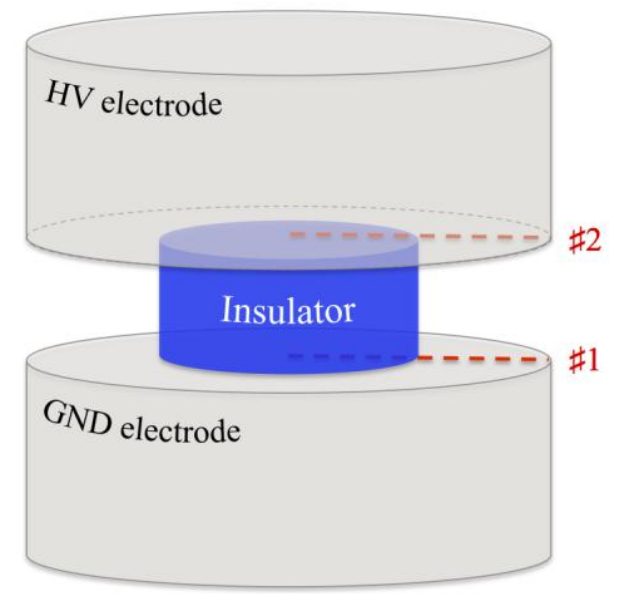

Figure 9. Schematic representation of the cylindrical electrodes/insulator configuration used for the calculation of the electric field strength. The electric field was computed at the electrode-insulator interface along the path \#1 (for the grounding GND electrode) and path \#2 (for the high-voltage HV electrode). When graded insulators were used, the particle-enriched region of the composite was placed in contact with the GND electrode.

Figure 10 shows the electric field strength along paths \#1 and \#2 for the pure epoxy and all investigated composites. The field strength at the epoxy-electrode interface was found to be equal to $42 \mathrm{kV} / \mathrm{mm}$ for both electrodes. At a NP concentration of $2 \mathrm{vol} \%$ the electric field strength on the GND electrode surface was reduced by $\approx 25 \%$ when a graded insulator was used rather than an uniform one, whereas the electric field strength on the HV electrode surface remained constant. At 6 vol\% the electric field strength on the GND electrode surface was reduced by $\approx 45 \%$ when a graded insulator was used rather than a uniform one, whereas it was increased by $\approx 55 \%$ on the HV electrode surface. A similar behavior was found for the nanocomposites containing $12 \mathrm{vol} \%$ of NP. In this case, the electric field strength on the GND electrode surface was reduced by $\approx 8 \%$ when a graded insulator was used rather than a uniform one, whereas on the HV electrode surface it was increased by the same relative amount.

In order to interpret the obtained results, attention has to be paid on the definition of the electric field $E$ for a fixed applied potential $V$. The electric field $E$ between two parallel plates 

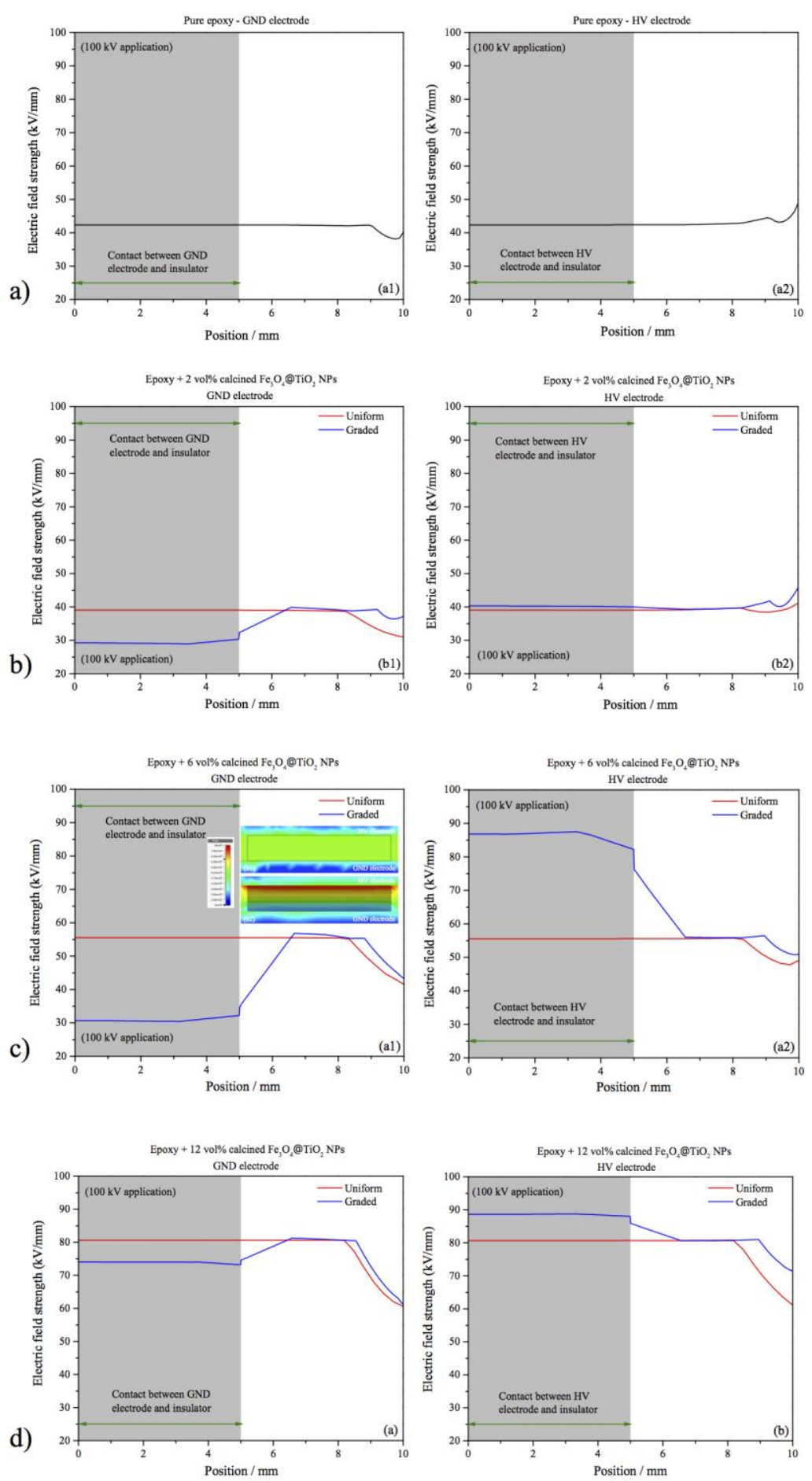

Figure 10. Electric field strength as a function of the position along the interface between the grounding (GND, left column) or high-voltage (HV, right column) electrodes and the pure epoxy (thickness $2.4 \mathrm{~mm}$, a) and homogeneous and graded composites with calcined $\mathrm{Fe}_{3} \mathrm{O}_{4} @ \mathrm{TiO}_{2}$ nanoparticles at concentrations of 2 vol\% (thickness $2.6 \mathrm{~mm}, \mathrm{~b}$ ), 6 vol\% (thickness $1.8 \mathrm{~mm}, \mathrm{c}$ ) and $12 \mathrm{vol} \%$ (thickness $1.3 \mathrm{~mm}, \mathrm{~d}$ ). The inset in Figure 10b shows the electric field strength distribution inside the homogeneous insulator with 6 vol\% of nanoparticles (top) or the graded one (bottom) and in part of the two electrodes. 
is defined as the ratio of $V$ to the distance $d$ between the plates and does not depend on the relative permittivity $\varepsilon_{r}$ of the materials used as a dielectric. If the material is homogeneous, the voltage changes linearly along $d$. On the contrary, the voltage drop across a graded permittivity dielectric depends on the charges $Q$ in each layer as well as on its capacitance $C$, since $\mathrm{V}=\mathrm{Q} / \mathrm{C}$. Given that the capacitance depends on the relative permittivity $\varepsilon_{r}$ as $\mathrm{C}=\varepsilon_{r} \varepsilon_{0} A / d$, where $\varepsilon_{0}$ is the permittivity of vacuum and $A$ is the area of the dielectric in contact with the electrodes, an increase in $\varepsilon_{r}$ leads to a decrease in the electric field $E$ if the total charge $Q$ remains constant. Unlike potential, charge cannot however be kept constant. Therefore, the behavior exhibited by the different graded composites cannot be explained merely in light of their relative permittivities in the interfacial regions, but rather relies on a more complex interplay between $\varepsilon_{r}$ and $Q$, which in turn depends on the morphology of the gradient. Nonetheless, it can be inferred that a reduction of the electric field at the interface between the electrode and the insulator can be attained having a decreasing gradient of permittivity going from the surface of the electrode towards the center of the insulator. The results reported clearly indicate how the proposed synthetic strategy, making use of magnetic fields, can be employed towards the synthesis of $\varepsilon$-FGM. Indeed, steep permittivity gradients can be generated as a result of the creation of nanoparticle concentration gradients as well as of the alignment of such nanoparticles in high aspect-ratio structures. The electric field strength reduction generated when the permittivity of the insulator decreases when going from the electrode surface towards the center of the dielectric suggests that $\varepsilon$-FGM with a Ushape permittivity would simultaneously generate a beneficial effect on both the electrode surfaces and would constitute a better alternative to homogeneous nanocomposite insulators in terms of durability and insulation performance. Nanocomposites with a U-shape permittivity could be synthesized through the application of the magnetic field generated by two magnets in repulsion configuration. Indeed, the exact placement of the nanocomposite in 
the middle of the two permanent magnets would lead to the motion of the nanoparticles towards the two sides of the composite, giving rise to the desired gradient.

\section{Conclusions}

Graded permittivity composites ( $\varepsilon$-FGM) were synthesized through the application of an external magnetic field to suspensions of $\mathrm{Fe}_{3} \mathrm{O}_{4} @ \mathrm{TiO}_{2}$ nanoparticles with a relative permittivity of 31 in a thermosetting epoxy resin. The application of the magnetic force not only induced the movement of the particles, but also caused their alignment in high aspect ratio structures, resulting in rather steep permittivity gradients. Numerical simulations clarified how the graded composites, employed as insulators, have the potential to efficiently reduce the electric field strength on the electrode surface in contact with the particleconcentrated region of the insulator, up to $45 \%$ compared with homogeneous analogues. The results reported constitute a good basis for the design of graded permittivity materials with remarkable properties to improve the lifetime of electrical power equipment.

\section{Acknowledgements}

The authors are indebted to the Swiss National Science Foundation (SNF Project 200020$155888 / 1)$ for financial support of this work.

\section{References}

[1] Tanaka T, Okamoto T, Nakanishi K, Miyamoto T. Aging and related phenomena in modern electric power systems. IEEE Trans Dielectr Electr Insul. 1993;28:826-44. 
[2] Kurimoto M, Kai A, Kato K, Okubo H. Fabrication of permittivity graded materials for reducing electric stress on electrode surface. IEEE Int Sympos Electr Insul. 2008:265-8.

[3] Kurimoto M, Kato K, Hanai M, Hoshina Y, Takei M, Okubo H. Application of functionally graded material for reducing electric field on electrode and spacer interface. IEEE Trans Diel Electr Insul. 2010;17:256-63.

[4] Nardi T, Leterrier Y, Karimi A, Månson J-AE. A novel synthetic strategy for bioinspired functionally graded nanocomposites employing magnetic field gradients. RSC Adv. 2014;4:7246-55.

[5] Nardi T, Leterrier Y, Månson J-AE. bioinspired functionally graded nanocomposites synthesized through magnetophoretic processes for tailored stress reduction. MRS Proc: Cambridge Journals Online; 2014.

[6] Nardi T, Canal LP, Hausmann M, Dujonc F, Michaud V, Månson J-AE, Leterrier Y. Stress reduction mechanisms during photopolymerization of functionally graded polymer nanocomposite coatings. Prog Org Coat. 2015;87:204-12.

[7] Bumb A, Brechbiel MW, Choyke PL, Fugger L, Eggeman A, Prabhakaran D. Synthesis and characterization of ultra-small superparamagnetic iron oxide nanoparticles thinly coated with silica. Nanotechnology. 2008;19:335601-6.

[8] Buscaglia MT, Buscaglia V, Curecheriu L, Postolache P, Mitoseriu L, Ianculescu AC, Vasile BS, Zhe Z, Nanni P. $\mathrm{Fe}_{2} \mathrm{O}_{3} @ \mathrm{BaTiO}_{3}$ core-shell particles as reactive precursors for the preparation ofmultifunctional composites containing different magnetic phases. Chem Mater. 2010;22:4740-8.

[9] Leite Vasconcelos DC, Costa VC, Martins Nunes EH, Soares Sabioni AC, Gasparon M, Vasconcelos WL. Infrared spectroscopy of titania sol-gel coatings on 316L stainless steel. Mater Sci Appl. 2011;2:1375-82.

[10] Cullity BD, Graham CD. Introduction to magnetic materials: John Wiley \& Sons, NewYork; 2009.

[11] Nardi T. Functionally graded polymer nanocomposites using magnetic fields. $\mathrm{PhD}$ thesis, 2015:EPFL \#6711.

[12] Phu ND, Ngo DT, Hoang LH, Luong NH, Chau N, Hai NH. Crystallization process and magnetic properties of amorphous iron oxide nanoparticles. J Phys D: Appl Phys. 2011;44:1-15.

[13] Kim JY, Jung HS, No JH, Kim JR, Hong KS. Influence of anatase-rutile phase transformation on dielectric of sol-gel derived $\mathrm{TiO}_{2}$ thin film. J Electroceram. 2006;16:447-51. 
[14] Ansar MZ, Atiq K, Alamgir S, Nadeem S. Frequency and temperature dependent dielectric response of $\mathrm{Fe}_{3} \mathrm{O}_{4}$ nano-crystallites. J Sci Res. 2014;6:399-406.

[15] Koumoto K, Masuda Y, Wang DJ. Biomimetic micropatterning of titanium dioxide thin films for gate dielectrics. Int J Soc Mater Eng Resour. 2002;10:49-52.

[16] Maxwell-Garnett JC. Colours in metal glasses and metal films. Phil Trans R Soc London. 1904;3:385-420.

[17] Bruggemann DAG. Berechnung verschiedener physikalischer konstanten von heterogenen substanzen. I. Dielektrizitätskonstanten und leitfähaigkeiten der mischkörper aus isotropen substanzen. Annalen der Physik. 1935;416:636-64.

[18] McLachlan DS, Priou A, Chernie I, Isaac E, Henry E. Modeling the permittivity of composite materials with general effective medium equation. J Electromag Wave. 1992;6:1099-131.

[19] Youngs IJ. Exploring the universal nature of electrical percolation exponents by genetic algorithm fitting with general effective medium theory. J Phys D: Appl Phys. 2002;35:3127-37.

[20] Koledintseva M, Ravva PC, Dubroff R, Drewniak J, Rozanov K, Archanbeault B. Engineering of composite media for shields at microwave frequencies. Electromag compat. 2005;1:169-74.

[21] Koledintseva MY, Ravva PC, Huang JY, Drewniak JL, Sabirov M, Bodrov VV, Sourkova IV, Sourkov VI. Power absorption of near field of elementary radiators in proximity of a composite layer. Prog Electromag Res Sympos. 2006:15-21.

[22] Jebbor N, Bri S. Effective permittivity of periodic composite materials: numerical modeling by the finite element method. J Electrostat. 2012;70:393-9. 
Table 1: Quantification of the different crystalline phases as well as of the amorphous phase of $\mathrm{Fe}_{3} \mathrm{O}_{4} @ \mathrm{TiO}_{2}$ nanoparticles. The sample indicated with $\mathrm{Fe}_{3} \mathrm{O}_{4} @ \mathrm{TiO}_{2}-\mathrm{C}$ was subjected to calcination. All quantities are expressed in weight $\%$.

\begin{tabular}{|l|c|c|c|c|}
\hline Nanoparticle type & Rutile & Anatase & Crystalline & Amorphous \\
& & & Magnetite & \\
\hline $\mathrm{Fe}_{3} \mathrm{O}_{4}$ & 0 & 0 & $51.44 \pm 0.29$ & $48.56 \pm 0.61$ \\
\hline $\mathrm{Fe}_{3} \mathrm{O}_{4} @ \mathrm{TiO}_{2}$ & 0 & 0 & $19.77 \pm 0.28$ & $80.23 \pm 0.83$ \\
\hline $\mathrm{Fe}_{3} \mathrm{O}_{4} @ \mathrm{TiO}_{2}-\mathrm{C}$ & 0 & $35.18 \pm 0.20$ & $32.58 \pm 0.23$ & $32.23 \pm 0.50$ \\
\hline
\end{tabular}

